\title{
A Sound Source Tracking Method of Distributed Microphone Array
}

\author{
Huannan Lin and Yiwen Zhang \\ Department of Information Engineering, Engineering College of the Chinese Armed Police Force, Xi'an, Shaanxi, China
}

\begin{abstract}
Distributed microphone array is composed of multiple spatial random microphone array, and this kind of way avoid the constraints of the topology of traditional microphone array, and it can perform a flexible positioning and application. Based on this, in this paper, we analyze the characteristics of the array, especially in the process of signal processing, we show the key technologies such as time synchronization, data fusion, and proposed the sound source tracing method under the practical acoustic environment .with the help of simulation experiment, we establish a model, and put forward a distributed interactive multiple model particle filter tracking algorithm, which has an important theoretical significance to improve tracking precision.
\end{abstract}

Keywords-distributed array microphones; sound source-tracking; time dalay

\section{INTRODUCTION}

The microphone is the sensor that pick up sound signal. The survey that pick up the microphone sound signal can show that ambient noise and reverberation effect is rather large effect on the single microphone radio performance. Distributed microphone array for the spatial filtering characteristics, compared with single microphone, in suppressing noise and its effect of reverberation interference effect is better. However, there are a lot of loopholes of sound source-tracking method theory and practical skills of operation of distributed microphone array, which need our technology researchers to improve perfect lately.

\section{THE CHARACTERISTICS AND THE CORE TECHNOLOGY}

Distributed microphone array have more than one microphone order together, it gathered many advantages that the single microphone does not have, thus it has a wide application prospect.

\section{A. The Characteristics of Distributed Microphone Array}

Compared with the traditional microphone array, the distributed microphone array is no rule of topological structure, so its performance has an increasingly diversified development. The space position of Array is also putting more flexible. Moreover, different from the traditional microphone arrays, even damage to individual array does not affect the network performance. Distributed microphone array structure has the characteristics of diversity and irregularity, so the distributed microphone array signal processing method has the better characteristics of universality than the traditional microphone array in dealing with issues.

The element spacing of distributed microphone array must meet space sampling theorem. In order to avoid the spatial aliasing for sampling signal, distributed microphone array element spacing must be under effective control and ensured the length of the array element spacing less than or equal to sampling signal wavelength. Of course to the array element spacing problem about the specific distributed microphone array, we still need to according to the specific application of the actual situation.

\section{B. Distributed Microphone Array of Core Technology}

On view in numerous distributed microphone array, if all arrays are on the location and tracking that will bring to sound source-tracking talent a large amount of work. So, choosing the appropriate number of microphone array application to participate in the distributed problem solving will improve the speed of distributed microphone array system, promote the data fusion and make the technical guarantee of the sound source tracking more effectively. The early source tracking method of distributed microphone array is that once a source close it and the target array detect the signal, then the target began to component dynamic cluster.

Figure I illustrate distributed processing where every node estimates its local source location model parameters using the tracking method.

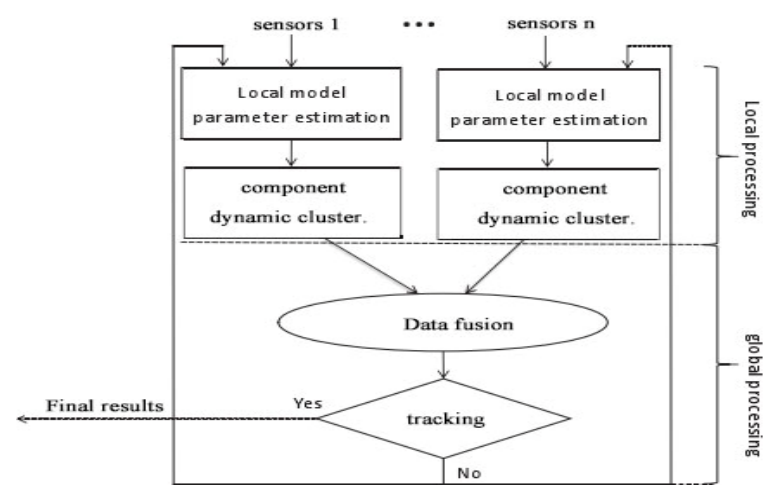

FIGURE I. THE MODEL OF TRACKING METHOD

Time synchronization is another important supporting technology. The requirements come from the two module: one is when in the source tracking process the generalized cross-correlation algorithm calculate time delay and the tracking interactive multiple model particle filter algorithm of sound source target must be consistent, otherwise it will cause big error to the distributed microphone array.

In the whole process of sound source-tracking, each array will have a following result. How to get multiple data together effectively to solute the problems of distributed microphone array together in the application process is the most critical steps that the data fusion in the data information processing of microphone array. In the data fusion process of distributed 
microphone array, we need fusion processing with each array data according to the certain logic algorithm, in order to obtain the most accurate results.

Table1show the weighting function that including measurement (Frames), mean, and deviation in order to show the significance of the method.

TABLE I. WEIGHTING FUNCTION

\begin{tabular}{ccc}
\hline $\begin{array}{c}\text { Weighting } \\
\text { Function }\end{array}$ & $\begin{array}{c}\text { No. of } \\
\text { (Frames) } \\
\text { (Frame }\end{array}$ & $\begin{array}{c}\text { Mean } \\
\text { (Deg.) }\end{array}$ \\
\hline Without & 404 & 3.346 \\
ML & 464 & 1.774 \\
PHAT-GCC & 490 & 1.298 \\
SRP & 496 & 0.871 \\
\hline
\end{tabular}

\section{THE SOUnd SOURCE LOCATION TRACKING}

\section{A. Tracking Location of Sound Source Based on Fingerprint}

So far there have many algorithm including controlled beam forming and sampling signal arrival time difference, space spectrum and so on. The sound source location tracking method above belongs to the direct calculation method, and another matching locating method of source tracking is the basis calculation on database. This method is more complex than the direct calculation method, it need to collect and calculate some prior data. Then establish a database to store it up and dealing with the received sampling signal and the existing data on database together in the processing of sound source tracking location so that it can through some internal connection between them to calculate the location of the target source. The sound source method of Distributed tracking microphone array determine the fingerprint orientation according to the position of the signal source. Once deployed the network environment of sound source tracking of distributed microphone array, it will determine the data from the collecting point of the fingerprint to and its corresponding position according to the demand of the network environment. The mobile signal source have an process of collection and processing for signs of each patch. The signals of each patch and the received signal characteristics and collected coordinates of each receiver are set as a group of data.

Figure II illustrate the whole process of sound source tracking that based on fingerprint.

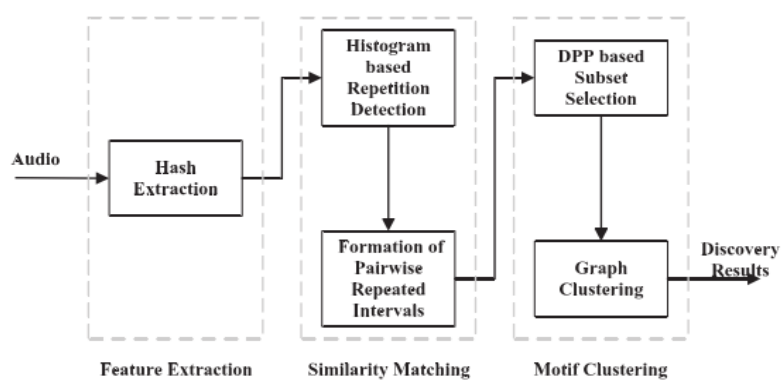

FIGURE II. THE ARCHITECTURE OF OUR FINGERPRINT MOTIF DISCOVERYSYSTEM.
The tracking location technology of sound source based on fingerprint is introduced into the distributed microphone array, finally realizes the sound source location. Sound track signal, however, is not the same as general of rf signal, distributed microphone array is not equal to the wireless network too. If it want to have a sound source tracking of distributed microphone array then two big problems including fingerprint parameters and background noise need to be solved.

\section{B. Method of Sound Source Tracking Location}

Generally speaking, previous deterministic method mainly involves three methods namely, nearest neighbor method, $\mathrm{K}$ nearest neighbor method and the weighted $\mathrm{K}$ nearest neighbor method. As the development of computer technology, the traditional deterministic method also has a certain improvement With the aid of improvement of the $\mathrm{K}$ nearest neighbor method or the weighted $\mathrm{K}$ nearest neighbor method, even if the sound source-tracking position of distributed microphone array not in the database, it can also have a good locating performance. Of course, the accuracy of this kind of sound source position tracking method in the database will be down. We specifically have a processing and improvement to it. In short, we set a distance threshold value $\theta 1$ of vector theta. If the smallest distance between the observation vector and the existing vector of distributed microphone array is smaller than the threshold, then we can use the nearest neighbor method to realize the sound source tracking. We using weighted K neighbor method.

\section{PERformance \& EVAlution}

\section{A. Sound Localization System}

In this experiment, the application of voice simulation is matlabR2015b platform .In order to change the sampling frequency, we set up the position of sound source in the three dimensional space is $X=(x, y, z)$ and the receiving position of distributed microphone array is $X^{\prime}=\left(x^{\prime}, y^{\prime}, z^{\prime}\right)$. Since that the distance between sound track position and distributed microphone array is $R=\left|x-x^{\prime}\right|$, f represent the frequency sampling data, $\omega=2 \pi f, c=340 \mathrm{~m} / \mathrm{s}$ indicates the spread speed of sound in air.

Figure III illustrate the simulated environment that in a small house with 7 meters wide and 8 meters long. We have 8 microphone that put in a distributed way.

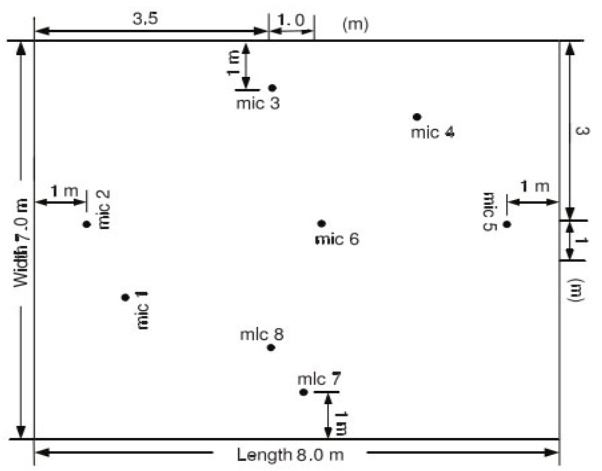

FIGURE III. SIMULATED ENVIRONMENT 


\section{B. Signal Preprocessing}

In the process of experiment, we have a $16 \mathrm{KH}$ sampling for speech signal and divide the wavelength of sampling signal of the whole distributed microphone array into 1024 frames. In short, the specific length of time is $64 \mathrm{~ms}$. We add an window to signal frame. That is divide the microphone into group which a set of two. Then the $\mathrm{n}$ group signal of microphone positioning $X_{i}$ in time ${ }^{t}$. Finally we calculate the time delay.

On the basis of the signal model, the generalized algorithm, analysis of the observation equation and distributed interactive multiple model particle filter, the sound source tracking algorithm can be simply described as:

To set Kas the total length of simulation, $M$ as the number of motion model, $\mathrm{S}$ as the number of array in the distributed microphone array,

Input: $X_{0}^{r}=X_{0}, P_{0}^{r}=\Pi_{0}>0, \mu_{0}^{r}, \mathrm{r}=1,2,3, \ldots \ldots, \mathrm{M}$.

For $\mathrm{k}=1$ to $\mathrm{K}$ within the cluster head and cluster member nodes will, in turn, complete the following calculation:

(1)Within the cluster node s the delay estimation $\tau_{k}^{s}$ can be got through calculating generalized algorithm,

(2)Calculate the model prediction probability $\mu_{k-1}^{r / m}$

(3)The model $\mathrm{R}$ in the state of $\mathrm{k}$ - 1 time $X_{k-1}^{r, 0}$ and the state covariance $P_{k-1}^{r / m}$,

(4)Calculate the update model probability $\mu_{k}^{r}$

(5)Get the global estimate $X_{k}^{r}$ of the cluster model $\mathrm{r}$ in current state and the corresponding covariance $P_{k}^{r}$

(6)Integrate the data and motions get the sound source motion state $X_{k}$ in time $\mathrm{K}$ and Covariance matrix $P_{k}$ input: $X_{k}, P_{k}, k=1,2,3 \ldots \ldots, k$,

The simulation formation show as Figure IV:

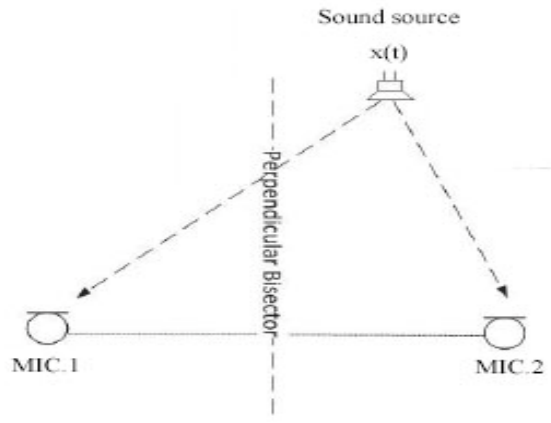

FIGURE IV. SIMULATED FORMATION.

To accurately calculate time delay in the above steps, each groups need to run an independent particle filter. At the time of initialization, we can assume that the prior probability is $p\left(x_{0}\right)=0.6$. The data fusion is calculated by using the Weighted summation. The whole simulation process and the final result is shown in Figure IV.

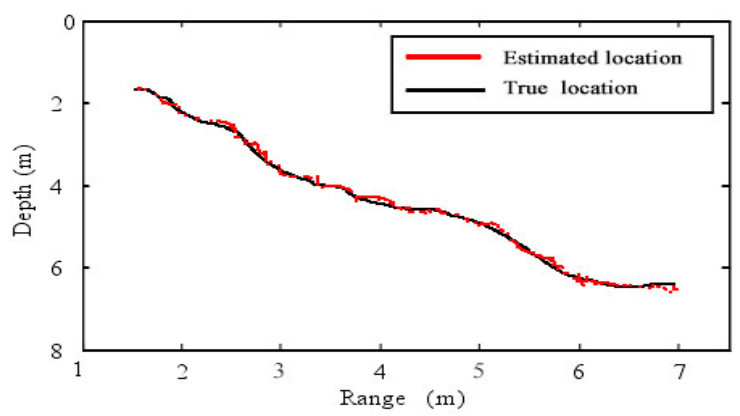

FIGURE V. SIMULATION PROCESS

\section{CONCLUSIONS}

Distributed microphone array is the combination of multiple microphone application. Compared with single microphone, it has a good effect on suppress noise and reverberation interference effect. What's more, distributed microphone array can put the position flexibly in space so that the specific sound source-tracking won't be affected by the placement. Distributed microphone array spacing, of course, must satisfy the space sampling theorem, to be effective computing time delay. Currently, researchers carry on the emulational results and have some good experiment results. But for the sound source tracing method of distributed microphone array, continuous innovation and improvement is needed.

\section{REFERENCES}

[1] N. Patwari, J. N. Ash, S. Kyperountas, A. O. Hero III, R. L.Moses, and N. S. Correal, "Locating the nodes: cooperativelocalization in wireless sensor networks,” IEEE Signal Process. Mag., vol. 22, no. 4, pp. 54 - 69 , July 2005.

[2] J. N. Ash and R. L. Moses, Self-localization of sensor networks, chapter 13, pp. 409 - 437, In Haykin and Liu ,2009.

[3] N. Ono, H. Kohno, N. Ito, and S. Sagayama, "Blind alignmentof asynchronously recorded signals for distributed microphonearray,” in Proc. IEEE Workshop on Applications of Signal Processing to Audio and Acoustics, Oct. 2009, pp. 161-164.

[4] Mc Cowan, M. Lincoln, and I. Himawan, "Microphone arrayshape calibration in diffuse noise fields,” IEEE Trans. Audio,Speech, Lang. Process., vol. 16, no. 3, pp. 666 - 670, Mar.2008.

[5] Z. Liu, Z. Zhang, L.-W. He, and P. Chou, "Energy-based soundsource localization and gain normalization for ad hoc microphone arrays," in Proc. IEEE Intl. Conf. on Acoust., Speech,Signal Process. (ICASSP), 2007, pp. II-761-II-764.

[6] C. Hue, J. P. Le Cadre, and P. Perez, "Sequential Monte Carlo methods for multiple target tracking and data fusion,” IEEETrans. Signal Process. vol. 50, no. 2, pp. 309-325, Feb. 2002.

[7] M. Chen, Z. Liu, L.-W. He, P. Chou, and Z. Zhang, "Energy-based position estimation of microphones and speakers for ad-hoc microphone arrays,” in Proc. IEEE Workshop on Applications of Signal Processing to Audio and Acoustics, 2007.

[8] Y. Kuang and K. Astrom, "Stratified sensor network self-calibration from TDOA measurements," in Proc. European SignalProcess. Conf., Marrakech, Morroco, 2013, pp. 1-5. 
[9] Markovsky, Low Rank Approximation: Algorithms, Implementation, Applications, Springer, 2012.

[10] L. D. Stone, R. L. Streit, T. L. Corwin, and K. L. Bell, Bayesian Multiple Target Tracking, Boston, USA: Artech House,2013.

[11] P. D. Jager and A. Hashemi-Sakhatsari, “Automatic microphone array position calibration using an acoustic soundingsource," in Proc. IEEE Conf. Industrial Electronics and Applications (ICIEA), May 2009, pp. $2110-2113$.

[12] M. Crocco, A. Del Bue, M. Bustreo, and V. Murino, “A closedform solution to the microphone position self-calibration problem," in Proc. IEEE Intl. Conf. on Acoust., Speech, SignalProcess. (ICASSP), Mar. 2012, pp. 2597 - 2600.

[13] S. Zhayida, F. Andersson, Y. Kuang, and K. Astrom, "An automatic system for microphone self-localization using ambientsound,” in Proc. European Signal Process. Conf., Lisbon, Portugal, 2014, pp. 954-958.

[14] Y. Kuang, S. Burgess, A. Torstensson, and K. Astrom, “A complete characterization and solution to the microphoneposition self-calibration problem,” in Proc. IEEE Int. Conf. Acoust. Speech, Signal Process., Vancouver, Canada, 2013, pp.3875-3879. 